\title{
Lens-less bending and concentration of light by volume hologram
}

\author{
Mei-Li Hsieh, ${ }^{1,{ }^{\star}}$ Cheng-Te Peng, ${ }^{1}$ Hong -Yu Chen, ${ }^{1}$ and Shawn-Yu Lin ${ }^{2}$ \\ ${ }^{1}$ Department of Photonics, National Chiao-Tung University, Hsinchu, Taiwan \\ ${ }^{2}$ The Future Chips Constellation, Department of Physics, Applied Physics and Astronomy, \\ Rensselaer Polytechnic Institute, Troy, NY 12180, USA \\ *Corresponding author: mlh@cc.nctu.edu.tw
}

In this paper, a new approach is proposed to realize a lens-less and 90-degrees light-bending by a volume-holographic element, consisting of 3D sub-wavelength index-gratings throughout the sample. In this approach, a top incident plane-wave is diffracted by 90 -degree and guided into a planar volume hologram. Conversely, a side-incident light may be guided and diffracted out of the top sample surface. The diffraction-efficiency $\eta$ of light was studied in real-time during light-exposure and also in the dark after exposure to observe "dark-enhancement". It is shown that 90-degree light-bending at normal incidence can be achieved with a high efficiency, $\eta \sim 70 \%$, and a small angular-tolerance, $\Delta \theta=0.02^{0}$. It is further shown, as a proof-of-concept, that a 5-degree cylindrical-wave top-incident beam may be used to improve angular tolerance to $\Delta \theta=5^{0}$, but with a reduced efficiency of $\eta \sim 0.80 \%$.

Keywords: Holographic optical elements; Volume gratings; Diffractive optics. 
Holographic elements have been widely used in optical system to replace traditional optical devices. Examples are compact holographic lens, holographic grating, and holographic concentrator for solar energy harvesting [1-3]. One of the key issues of a holographic element concerns optical properties of the recording materials. However, PQ/PMMA materials has recently been shown to have superior optical characteristics, such as high contrast refractive-index variation, low shrinkage after exposure, environmentally stable and no need for lithographic development [4-7]. Currently, there is an emerging interest in using holographic elements for compact display system with edge-lit hologram [8-10] and for solar harvesting to achieve high conversion-efficiency and enable an easy adoption to a building's nonplanar structure. To achieve high conversion-efficiency, a transmission holographic element has been proposed to split solar radiation into regions according to the spectral sensitivity of the cells [11-13]. To enable adaption to building structure, several materials approaches have been developed that include the use of thin-film, polymer, or dye-sensitized photovoltaic cells. Another optical approach is to combine low-cost concentrators with high-efficiency solar cells $[14,15]$. So far, there is no holographic approach that utilizes 90-degree holographic recording geometry [16] to enable solar collection with a large collection angle, over the entire solar spectrum and with high concentration factor.

In this paper, we demonstrate lens-less bending and concentration of light by a volume holographic element (VHE). The PQ/PMMA material has a good optical quality and was chosen as the holographic recording material. The recording of index-grating was performed using two different schemes- top incident and 90-degree incident configurations. The efficiency of light-diffraction was studied in real-time and also in the dark to observe "dark-enhancement". We show that it is possible to achieve 90-deg light bending with high efficiency $\eta=70 \%$. Furthermore, this $90-$ deg bending scheme leads to a concentration factor of 2.5 times, without the use of any external optics. With further improvement in angular tolerance, this 90-degree light-bending scheme may be useful for compact and efficient solar harvesting.

We first study the optical properties of our PQ/PMMA materials, using a top-incident configuration for optical recording and reading. Figure 1(a) and (b) illustrate the recording and reading processes, respectively. In Figure 1(a), two plane-waves were incident from the sample-top with symmetric incident angle, 45 degrees. In Figure 1(b), a reading beam at the Bragg angle was incident from the sample-top, and the diffracted beam was retrieved. For this top-incident configuration, the recording and reading laser wavelengths were $\lambda=514.5 \mathrm{~nm}$ and $\lambda=632.8 \mathrm{~nm}$, respectively. The recording wavelength of $514.5 \mathrm{~nm}$ was chosen because of its relatively high absorption coefficient of $\alpha=2 \mathrm{~cm}^{-1}$ in our PQ/PMMA material [17]. Since the reading wavelength is different from the recording wavelength, the reading angle has to be modified in accordance with the Bragg condition [18] and is 60 degrees in air.

Figure 2(a) shows diffraction-efficiency $\eta$ v.s. exposure-energy measured in real time during light exposure. The testing was repeated for three samples of different thickness $\mathrm{d}=2,3,4 \mathrm{~mm}$, respectively. For $d=2 \mathrm{~mm}$ sample, its $\eta$ increases with increasing exposure energy, reaches a maximum 
of $\eta \sim 35 \%$ at $E^{\text {exposure }} \sim 4.2 \mathrm{~J} / \mathrm{cm}^{2}$ and eventually saturates at $\eta \sim 33 \%$. For the $\mathrm{d}=3 \mathrm{~mm}$ sample, its efficiency follows the same trend and exhibits a higher maximum value of $\eta \sim 51 \%$ at $\mathrm{E}^{\text {exposure }} \sim 3.9$ $\mathrm{J} / \mathrm{cm}^{2}$. The trend continues and the $\mathrm{d}=4 \mathrm{~mm}$ sample has an even higher maximum- $\eta$ of $\sim 71 \%$ at $\mathrm{E}^{\text {exposure }}$ $\sim 3.75 \mathrm{~J} / \mathrm{cm}^{2}$. This efficiency-characteristic is consistent with that predicted for a volume grating, namely, $\eta$ is proportional to the square of sin function of sample thickness, i.e., $\eta \propto \sin ^{2}(\kappa d)$ [18]. To first order, it follows that the maximum- $\eta$ should scale linearly with sample thickness $d$ as was observed. The agreement of our finding with theoretical prediction illustrates that our PQ-PMMA material is uniform and the recorded index-grating is well-defined. We next study change of $\eta$ of our PQ-PMMA as a function of time after light exposure and in the dark, i.e. dark time $t^{\text {dark }}$. We examine six samples of the same thickness, $\mathrm{d}=3 \mathrm{~mm}$, but with different exposure-energy $E^{\text {exposure }}=0.5,1.0,1.5,2.0,2.5$ and 4.0 $\mathrm{J} / \mathrm{cm}^{2}$, respectively. The data are shown in figure 2(b). For all six samples, their $\eta$ increases with the dark time initially (dark-enhancement), reaches a maximum value at $\mathrm{t}^{\text {dark }}=4 \sim 8$ hours and saturate at a steady-state value eventually. The optimum conditions are found to be $E^{\text {exposure }}=2.0 \mathrm{~J} / \mathrm{cm}^{2}$ and $\mathrm{t}^{\text {dark }}=6.8$ hours for a $d=3 \mathrm{~mm}$ PQ-PMMA sample, such that $\eta$ can reach a maximum value of $69 \%$ with a darkenhancement of 1.6 times.

Having established the optimum conditions, we now design a new volume-hologram system for solar collection/ concentration purpose. The new recording and reading procedure is called "90-deg incident configuration" and is shown in figure 1(c) and 1(d), respectively. In figure 1(c), one of the recording beams is incident from the top of sample and another from the left side. After recording, in figure 1(d), the reading beam is incident from the top and the diffracted beam would be guided along the planar volume hologram and exit from the right side of the sample. Thus, a top incident light is converted into a guided wave with its beam size determined by volume hologram thickness d. For example, let us assume that the size of our sample is $10 \times 5 \times 2 \mathrm{~mm}^{3}$, and the beam size from the top is $10 \times 5 \mathrm{~mm}^{2}$, then, the diffracted beam size from the side becomes $10 \times 2 \mathrm{~mm}^{2}$. Thus, the solar light with $10 \times 5 \mathrm{~mm}^{2}$ size incident from the top will yield a diffracted beam size of $10 \times 2 \mathrm{~mm}^{2}$, a concentration factor of 2.5 times. In other words, the diffracted or bent plane wave is narrower, as narrow as the recording plane wave in the holographic plate. Also, a solar-cell is to be placed at the side of the hologram to convert optical to electrical energy. In this 90-degree incident configuration, our proposed volume hologram approach is promising for achieving light-concentration without the use of any external optical element.

As the first attempt to realize the proposed 90-degree recording and reading concept, a two planewaves incidence setup was constructed and shown in Figure 3(a). Special care was taken to polish the two end faces of the PQ/PMMA material to be optically smooth. In the recording phase, two planewaves with $\lambda=514.5 \mathrm{~nm}$ were incident (green arrows) onto the PQ/PMMA sample. In the reading phase, a plane wave is incident on the top of the sample and the transmitted and diffracted waves measured. Figure 3(b) shows a photo of our system during the reading process. Light bending by 90 -degrees by a 
volume-hologram was clearly observed. Additionally, CCD photos were taken to better capture the transmitted and diffracted images and were shown in figure 3(c) and 3(d), respectively. The transmission beam is rather weak and has the same size as the incident beam, $5 \times 10 \mathrm{~mm}^{2}$. On the contrary, the intensity of the diffracted beam is strong, with a smaller beam size of $2 \times 10 \mathrm{~mm}^{2}$. By diffracting the incident light sideway, a concentration ratio of 2.5 times (or 250\%) is achieved. Hence, our holographic approach can give a high solar-concentration without the use of any external optics, offering an universal solution that can be integrated to any solar cells.

In Figure 4, we show quantitative measurements of diffraction-efficiency of our PQ/PMMA material in 90-deg recording configuration. Figure 4(a) shows the real-time diffraction- $\eta \square v s$. exposure energy for a sample of thickness $d=3 \mathrm{~mm}$. Here, reading beam of $\lambda=594.1 \mathrm{~nm}$ is used to minimize absorption loss of PQ/PMMA. Similar to that observed for the top-incident sample, the diffractionefficiency for the $90^{\circ}$ incident configuration increases initially with increasing $E^{\text {exposure }}$, reaches the maximum value of $\eta=50 \%$ at $E^{\text {exposure }} \approx 4.0 \mathrm{~J} / \mathrm{cm}^{2}$ and eventually saturates at $\eta=50 \%$ for $E^{\text {exposure }}>7$ $\mathrm{J} / \mathrm{cm}^{2}$. Figure 4(b) shows diffraction- $\eta$ v.s. dark-time $\left(t^{\text {dark }}\right)$ for eight samples of the same thickness $\mathrm{d}=3 \mathrm{~mm}$, but different $E^{\text {exposure }}=1.0,1.5,1.75,2.0,2.25,2.5,3.0$ and $4.0 \mathrm{~J} / \mathrm{cm}^{2}$, respectively. For the sample with $E^{\text {exposure }}=1.0 \mathrm{~J} / \mathrm{cm}^{2}$, its initial $-\eta$ is $20 \%$ and the maximum- $\eta 32 \%$ after $\sim 4$ hours dark-time. This corresponds to the low exposure regime, where index-grating is weak and diffraction-efficiency is low. The dark enhancement is found to be 1.7 times. For the sample with $E^{\text {exposure }}=1.75 \mathrm{~J} / \mathrm{cm}^{2}$, its indexgrating becomes stronger, and its initial- and maximum- $\eta$ are increased to $33 \%$ and $63 \%$, respectively, after 4 hours of dark time. The dark enhancement is found to be 1.9 times. The optimum condition was achieved for the sample with $E^{\text {exposure }}=2.25 \mathrm{~J} / \mathrm{cm}^{2}$, having an initial- and maximum- $\eta$ of $40 \%$ and $70 \%$, respectively, also after 4 hours of dark-time.

Figure 4(c) summaries the measured maximum and steady-state diffraction efficiency as a function of $E^{\text {exposure }}$. It is found that high diffraction- $\eta$ of $>65 \%$ can be achieved over a broad exposure energy range, $1.75 \leq E^{\text {exposure }} \leq 2.5 \mathrm{~J} / \mathrm{cm}^{2}$. The lower diffraction- $\eta$ at low exposure-energy, $E^{\text {exposure }} \leq 1.5 \mathrm{~J} / \mathrm{cm}^{2}$, may be due to a low index-grating in the PQ/PMMA volume hologram. For samples with $E^{\text {exposure }}>2.75 \mathrm{~J} / \mathrm{cm}^{2}$, the diffraction drops slightly below $60 \%$. One possible contributing factor to the efficiency drop may be caused by higher order scattering at higher exposure energy. We comment that further improvement in diffraction-efficiency to $>80 \%$ may be achieved by having a better uniformity of the PQ/PMMA material, as well as by polishing the end faces of PQ/PMMA to be more parallel to each other and to have optically smoother end faces.

To explain the performance of our 90-deg recorded volume-hologram, we calculate intensity distribution of the expected interference pattern. A detail schematic of our holographic recording is shown in Figure 5(a), where a plane wave is incident from the side $\left(\vec{k}_{1}\right)$ in the $\mathrm{x}$-direction. The top incident wave $\left(\overrightarrow{k_{2}}\right)$ can be either a planewave $\left(\theta=0^{0}\right)$ or a cylindrical wave $\left(\theta \neq 0^{0}\right)$, where $\theta$ is its angular 
extend. For $\theta=0^{0}$ case, the interference pattern is uniform along y-direction and is plotted in Figure 5(b) in the X-Z plane. The period $\Lambda$ and direction of the holographic grating is determined by $\overrightarrow{k_{1}}, \overrightarrow{k_{2}}$ and $\lambda$ of the two recording beams. From the plot, we find $\Lambda=368 \mathrm{~nm}$ and $\vec{K}=\overrightarrow{k_{1}}-\overrightarrow{k_{2}}$ to be along the $45^{0}$ direction (red arrow). Note that the pattern is independent of its location in the x-direction, meaning that only a single volume-grating is recorded throughout the sample. Using this grating pattern, a reading beam that matches Bragg condition of the grating can be efficiently diffracted and subsequently propagates along the $\mathrm{x}$-direction to the side. This calculation validates results of our 90-degree light-bending measurement shown in Fig. 3(b).

To motivate this work from solar application point-of-view, we consider a potential implementation of our VHE device using cylindrical-wave approach. The cylindrical wave is used to simulate the wide-angle incidence of sunlight. This is because a cylindrical wave may be decomposed, by Fourier transform, into many plane waves with a range of incident-angles. By doing so, we hope to increase angular-tolerance of our VHE. Furthermore, by increasing the $\theta$-tolerance, $\lambda$-tolerance would be increased accordingly[18]. As the first step, we calculate interference pattern using wide-angle cylindrical wave as the top incident beam. Our goal is to simultaneously record multiple gratings of different $\Lambda$ and directions inside the sample. Due to the use of cylindrical wave, the interference pattern is expected to be uniform only along $y$ - but not $\mathrm{x}$-direction. To illustrate this point, in Figure 5(c), we plot the computed pattern in the $\mathrm{x}-\mathrm{z}$ plane at three positions along $\mathrm{x}$ : (I) $\mathrm{x}=-2 \mathrm{~mm}, \mathrm{z}=0$; (II) $\mathrm{x}=0, \mathrm{z}=0$; (III) $\mathrm{x}=2 \mathrm{~mm}, \mathrm{z}=0$. Here, $\overrightarrow{k_{2}}$ spans an angular range of $\theta=\left(-45^{0},+45^{\circ}\right)$. Indeed, we find multiple gratings: $\Lambda=500 \mathrm{~nm}, 368 \mathrm{~nm}$ and $300 \mathrm{~nm}$ and $\vec{K}=\overrightarrow{k_{1}}-\overrightarrow{k_{2}}$ along the $59^{\circ}, 45^{\circ}$ and $30.9^{0}$ direction at different sample positions (I), (II) and (III), respectively. This result shows that our volume-hologram is capable of having a $\theta$-tolerance of $\Delta \theta=90^{\circ}$ and a $\lambda$-tolerance of $\Delta \lambda=200 \mathrm{~nm}$ [18]. As a result, after recording, a plane wave incident from the sample-top with a range of $\theta$ s and $\lambda$ s can be better matched to Bragg condition and diffracted to the side by approximately 90-degrees.

As a proof-of-concept of our cylindrical-wave approach, we perform $\theta$-tolerance measurement of our VHE device. The testing is done by mounting our VHE device on a rotational stage and measuring its 90 -degree diffraction-efficiency as a function of rotational angle $\theta$. As a reference for $\theta$-tolerance, we performed two additional recordings using a single plane-wave incident configuration at $\theta=0^{0}$ and two plane-waves incident configuration at $\theta=1$ and $-1^{0}$, i.e. angular multiplexing [19]. Figure 6(a) shows result for the single plane-wave configuration. The 90 -degree diffraction-efficiency and $\theta$-tolerance of are measured to be $\eta=58 \%$ and $\Delta \theta=0.02^{0}$, respectively. Figure 6(b) shows result of angular multiplexing. Two strong diffraction peaks of $\eta=53 \%$ were observed at two separate angles, $\theta=1$ and $-1^{0}$, confirming angular multiplexing. Yet, each diffraction peak still has a small $\theta$-tolerance of $\Delta \theta=0.02^{0}$. Figure $6(\mathrm{c})$ shows results of cylindrical-wave incidence with an angular extend of $5^{0}$ (i.e. edge-lit hologram by a cylindrical-wave). In this recording phase, cylindrical-wave was incident from the top (i.e. the larger 
face) and, in the reading phase, a plane-wave is incident to measure diffraction efficiency. Note that a broad $\theta$-tolerance of $\Delta \theta=5^{0}$ is observed, but at the expense of a lower efficiency of $\eta=0.8 \%$. The observed broad $\theta$-tolerance validates the proposed concept of using a cylindrical-wave to approximate wide angle incidence of sunray. The correspondingly reduced efficiency may be understood by an area argument. By examining the interference pattern of Figure 5(c), we notice that each region of the sample along $\mathrm{x}$-direction posses a different grating period/direction and is responsible for diffracting light from a specific angle-of-incidence. As a consequence, in our cylindrical-wave configuration, each sub-section of the sample area is responsible for diffracting light from a specific incident angle. Therefore, the effective area for light diffraction for each $\theta$ is reduced. This would explain the reduced diffraction efficiency. In our future work, we shall come up with a clever design to overcome this area-constrain by, perhaps, performing multiple exposures by shifting the exposure beams across the sample- spatial multiplexing. Nonetheless, this experiment is the first proof-of-concept experiment, illustrating that wider $\theta$-tolerance of our VHE can be accomplished using a cylindrical-wave approach.

Ultimately, the potential of a VHE as a solar-concentrator depends on the dynamic range of the hologram-material. Quantitatively, this material metric is characterized by the so called "M/\#" [20]. A typical M/\# for a 3mm thick PQ-PMMA hologram material is 3-5 [6]. In order to improve diffractionefficiency over broad angles for solar application, we believe M/\# of the chosen material should be improved by at least ten-fold. This is challenging. However, two technical points may be developed. First, one way to improve M/\# is to increase concentration of the photo-sensitive PQ-molecular or change its compositions. By modifying compositions, the material sensitivity and dynamic range for volume holographic recording can be improved [21]. Here, special process must be developed to ensure uniformity of PQ-molecular. Another factor of five may come from process refinement to produce more uniform hologram material, to synthesis a 2 3 times thicker material of 6-9 $\mathrm{mm}$ [6] and, hence, achieving a better efficiency and larger recording-capability.

In this paper, we propose a new technique to efficiently bend light by 90-degrees without the use of any external optical elements, such as lens and mirrors. This process of extreme light- bending is capable of collecting a large-area sunlight in a planar-incident fashion and converting it into a smaller area side emission, thus achieving compact solar energy concentration. In this experiment, a concentration factor of 2.5 times is demonstrated and a further improvement to 5-10 times is possible. Furthermore, our approach represents an universal scheme of light-collection and a subsequent sideemission and may be to integrated to high performance solar cells.

\section{ACKNOWLEDGMENTS}

S.Y.L. gratefully acknowledges financial support from DOE Office of Science under award DEFG02-06ER46347. MLH acknowledges financial support from MOST 103-2221-E-009-073 and also international travel support from DOE-BES. 


\section{REFERENCES}

1. D. H. Close, "Holographic Optical Elements", Optical Engineering, 14(5), 145402 (1975).

2. J. W. Goodman, Introduction to Fourier Optics, 2nd edition, Chap 9, p. 290, 1996.

3. J. E. Ludman, “Holographic solar concentrator”, Appl. Opt. 21(17), 3057-3058 (1982).

4. H. Franke, "Optical recording of refractive-index patterns in doped poly-methyl methacrylate films," Appl. Opt. 23, 2729-2733 (1984).

5. A. Pu and D. Psaltis, "High-density recording in photopholymer-based holographic threedimensional disks", Appl. Opt. 35(14), 2389-2398 (1996).

6. Ken Y. Hsu, Shiuan Huei Lin and Yi-Nan Hsiao, "Experimental characterization of phenanthrenequinoe-doped poly(methylmethacrylate) photopolymer for volume holographic storage", Opt. Eng. 42(5), 1390-1396 (2003).

7. H. Liu, D. Yu, X. Li, S. Luo, Y. Jiang, and X. Sun, "Diffusional enhancement of volume gratings as an optimized strategy for holographic memory in PQ-PMMA photopolymer", Opt. Express 18(7), 6447-6454 (2010).

8. L. H. Lin, “Edge-illuminated hologram”, J. Opt. Soc. Am. 60, 714A (1970).

9. J. Upatnieks, “Compact holographic sight”, J. Opt. Soc. Am. A 2, 13, p86 (1985).

10. Q. Huang and J. J. Caulfield, "Waveguide holography and its applications", in Practical Holography IV, S. A. Benton, ed., Proc. Soc. Photo-Opt. Instrum Eng. 1212, 149-157 (1990).

11. W. H. Bloss, M. Griesinger, and E. R. Reinhardt, "Dispersive concentrating systems based on transmission phase holograms for solar applications,” Appl. Opt. 21, 3739-3742 (1982).

12. A. G. Imenes and D. R. Mills, "Spectral beam splitting technology for increased conversion efficiency in solar concentrating systems: a review," Sol. Energy Mat. Sol. Cells 84, 19-69 (2004).

13. J. R. Riccobono and J. E. Ludman, Solar holography, in Holography for the New Millennium (Springer, 2002).

14. US Department of Energy, Office of Science, Basic Energy Needs for Solar Energy Utilization, April 18-21, 2005C.

15. M. Rogol et al., Solar Annual 2008, Photon Consulting. 
16. Y. Yang, A. Adibi, and D. Psaltis, "Comparison of transmission and the 90-degree holographic recording geometry”, Appl. Opt. 42(17), 3418-3427 (2003).

17. Mei-Li Hsieh, Wei-Cheng Chen, Hong-Yu Chen, and Shawn-Yu Lin, "Optimization of light diffraction-efficiency and its enhancement from a doped-PMMA volume holographic material", Opti. Commu. 308, 121-124 (2013).

18. H. Kogelnik, “Coupled wave theory for thick hologram gratings”, Bell Syst. Tech. J., 48, 2909-2947 (1969).

19. G. W. Burr, F. H. Mok and D. Psaltis, "Angle and space multiplexed holographic storage using the 90 geometry", Optics Communications, 117, 49-55 (1995).

20. F. Mok, G. Burr, and D. Psaltis, “A system metric for holographic memory systems,” Opt. Lett. 21, 886-888 (1996).

21.S. H. Lin, J. H. Lin, P. L. Chen, Y. N. Shiao and K. Y. Hsu, "Doped Poly(Methyl Methacrylate) Photopolymers for Holographic Data Storage,” Journal of Nonlinear Optical Physics \& Materials 15, 2, 239-252 (2006). 
Figure Caption:

Figure 1 Our volume hologram recording and reading schemes. (a), (b) Schematic of two-beam recording (green arrows) and reading (red arrows) procedures, respectively, for the top-incident configuration; (c), (d) Schematic of two-beam recording and reading procedure, respectively, for the 90deg incident configuration. The diffracted beam is directed along the planar volume hologram.

Figure 2 Optical characterization of diffraction-efficiency $\eta$ of the diffracted beam. (a) Real-time diffraction- $\eta$ vs. exposure energy for sample thickness $d=2,3$ and $4 \mathrm{~mm}$ for the top incident configuration. The maximum- $\eta$ is indicated by vertical arrows. A photo of the PQ/PMMA photosensitive material is shown in the inset. (b) The measured $\eta$ as a function of dark-time ( $t^{\text {dark }}$ ) for six samples of the same thickness $\mathrm{d}=3 \mathrm{~mm}$, but with different exposure-energy $E^{\text {exposure }}=0.5,1.0,1.5,2.0,2.5$ and $4.0 \mathrm{~J} / \mathrm{cm}^{2}$, respectively.

Figure 3 Testing setup and images of the transmitted and diffracted beams. (a) Our experimental setup for holographic recording and reading at 90-deg incidence. (b) A photo of light-bending by 90-degrees due to the build-in holographic grating. (c) and (d) are CCD images of the transmitted and diffracted light by our volume hologram. The diffracted beam exhibits 2.5 -times narrower beam-width and, hence, a 2.5-times light concentration.

Figure 4 Diffraction- $\eta$ of volume hologram for the 90-degreee incident configuration. (a) Real-time diffraction- $\eta$ vs. exposure energy for a sample of thickness $d=3 \mathrm{~mm}$. (b) Diffraction- $\eta$ v.s. dark-time ( $t^{\text {dark }}$ ) for eight samples of the same thickness $\mathrm{d}=3 \mathrm{~mm}$, but different exposure-energy $E^{\text {exposure }}=1.0,1.5$, $1.75,2.0,2.25,2.5,3.0$ and $4.0 \mathrm{~J} / \mathrm{cm}^{2}$, respectively. (c) A summary of the maximum and steady-state diffraction- $\eta$ vs exposure-energy.

Figure 5 Description and modeling of two-beam recording via 90-deg incident configuration. (a) A planewave is incident from the side along the $\mathrm{x}$-direction $\left(\mathrm{k}_{1}\right)$; Another planewave $\left(\theta=0^{0}\right)$ or cylindrical wave $\left(\theta \neq 0^{0}\right)$ is incident from the top. Here, $\theta$ is the angular extend of the top incident beam. (b) grating intensity profile in the $\mathrm{x}-\mathrm{z}$ plane for $\theta=0^{0}$ at three positions along $\mathrm{x}$ : (I) $\mathrm{x}=-2 \mathrm{~mm}, \mathrm{z}=0$; (II) $\mathrm{x}=0, \mathrm{z}=0$; (III) $\mathrm{x}=2 \mathrm{~mm}, \mathrm{z}=0$; (c) grating intensity profile in the $\mathrm{x}-\mathrm{z}$ plane for $\theta=90^{\circ}$ at three positions along $\mathrm{x}$ : (I) $\mathrm{x}=-2 \mathrm{~mm}, \mathrm{z}=0$ ); (II) $\mathrm{x}=0, \mathrm{z}=0$; (III) $\mathrm{x}=2 \mathrm{~mm}, \mathrm{z}=0$.

Figure 6 Angular-tolerance for the 90-degreee incident configuration. (a) Single plane-wave configuration at normal incidence; (b) Two plane-waves incident configuration at $\theta=1$ and $-1^{0}$, i.e. angular multiplexing; (c) Cylindrical-wave incident configuration. 
Figures

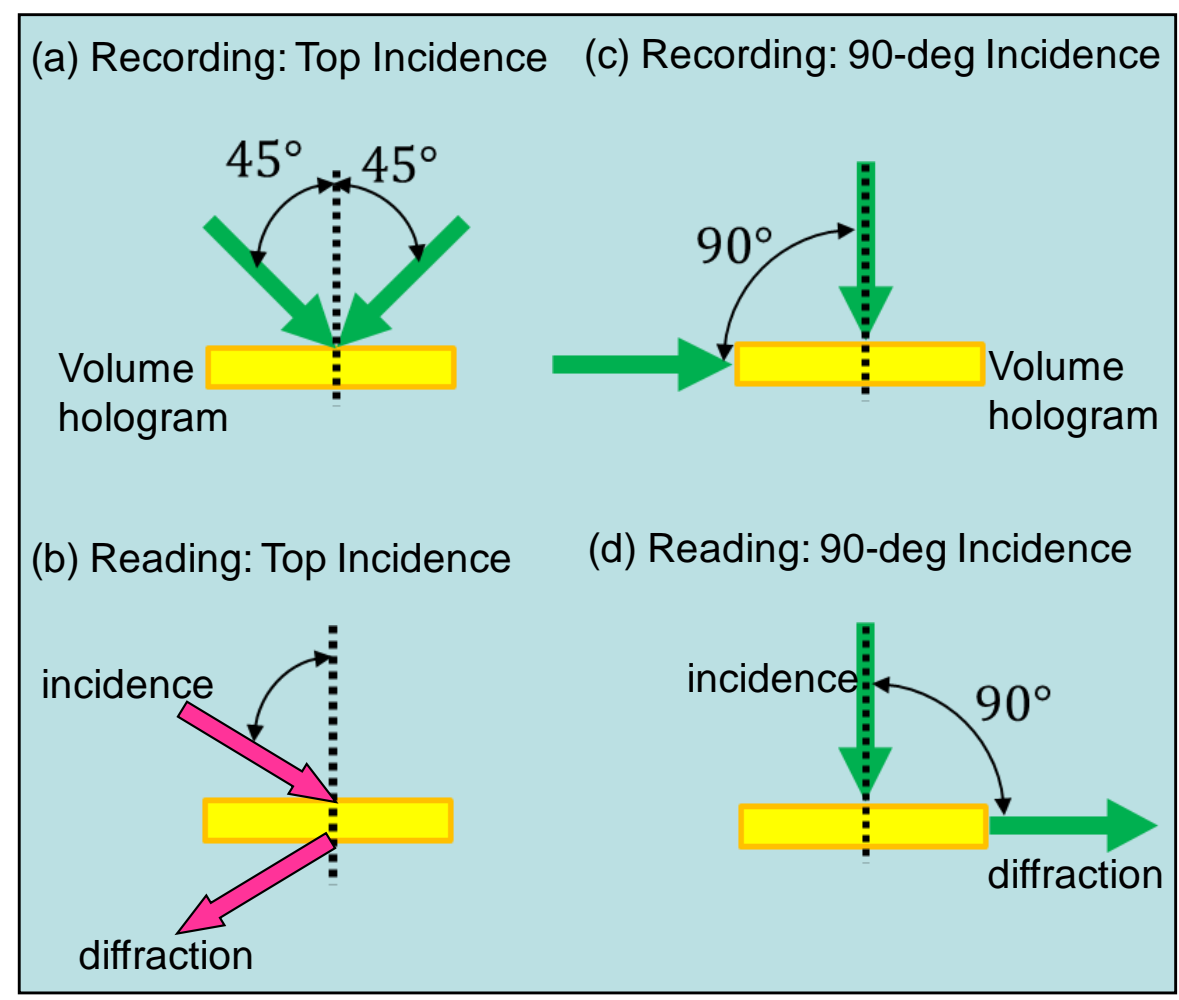

Figure 1 
(a)

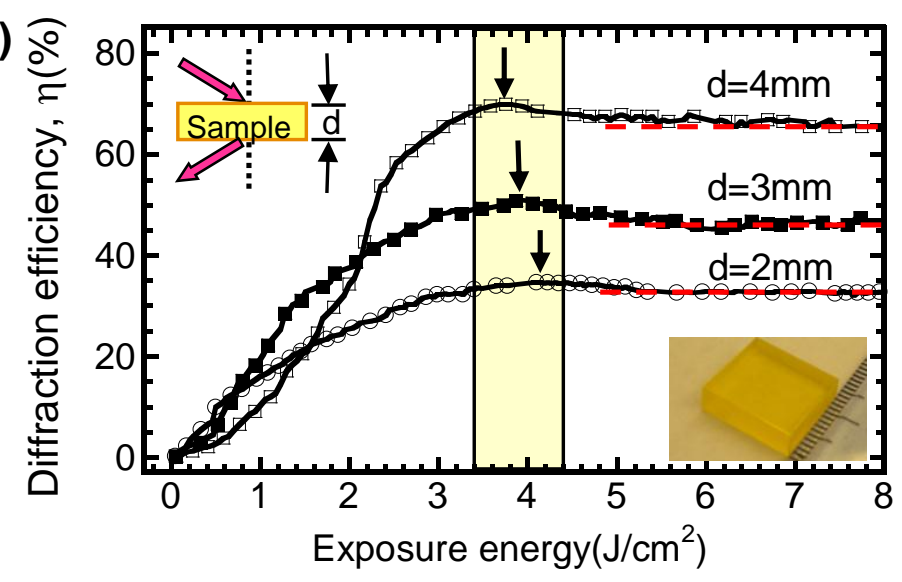

(b)

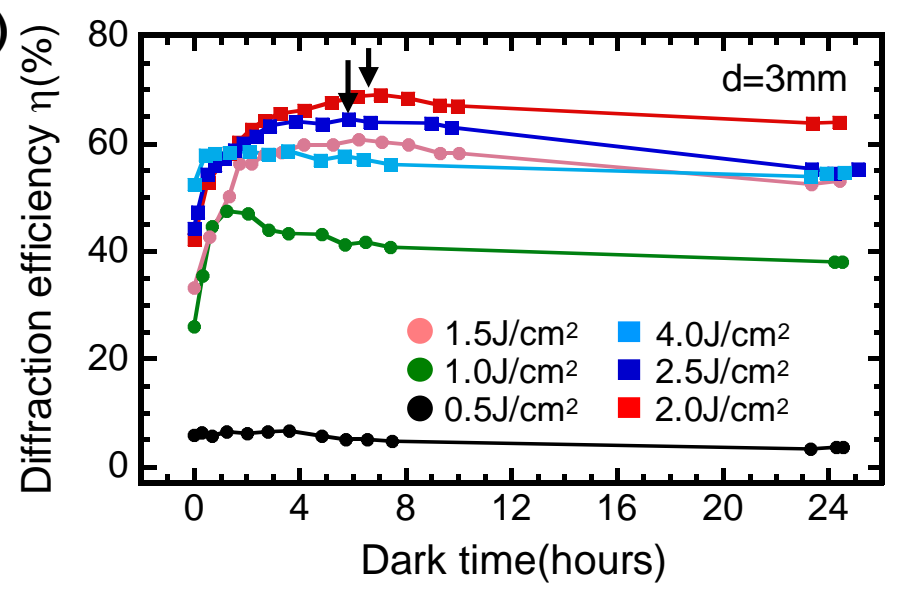

Figure 2 
(a) Setup for Optical Recording and Reading
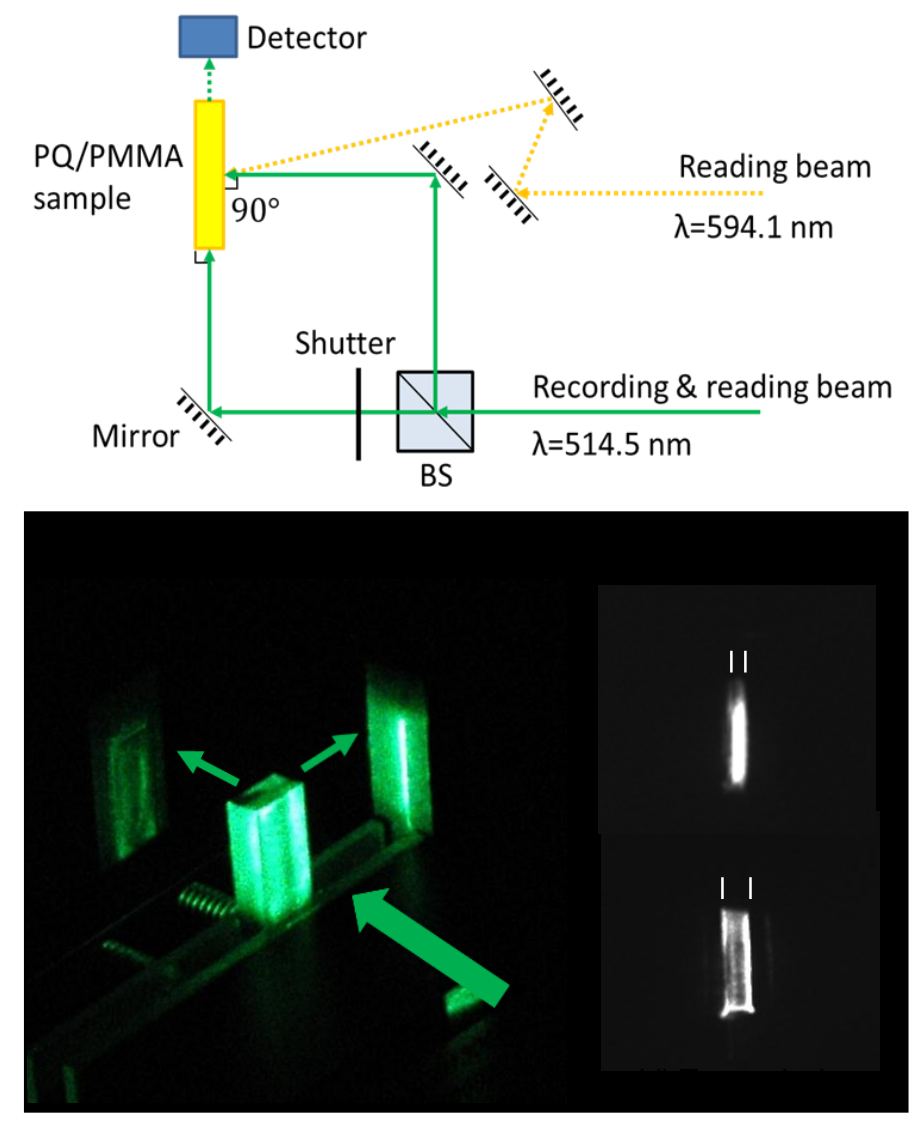

Figure 3 
(a)

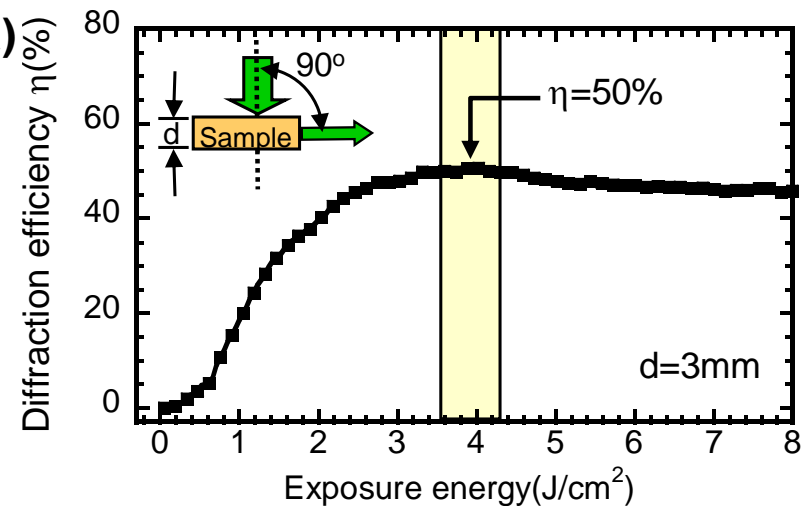

(b)

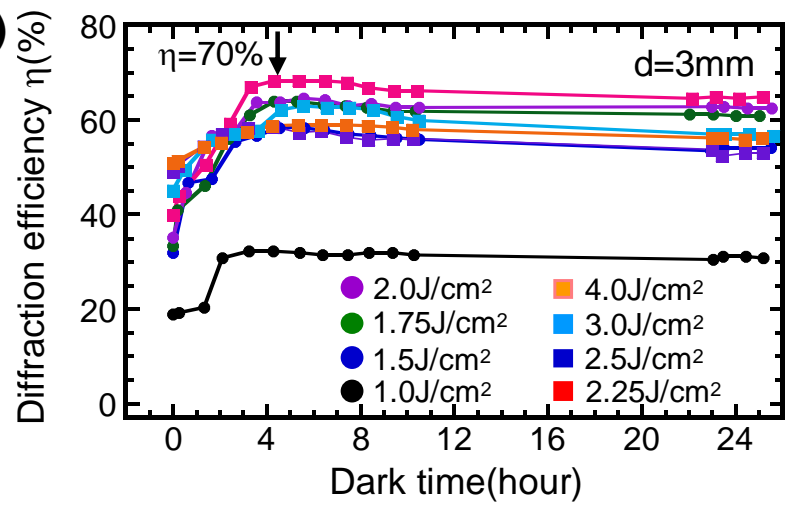

(c)

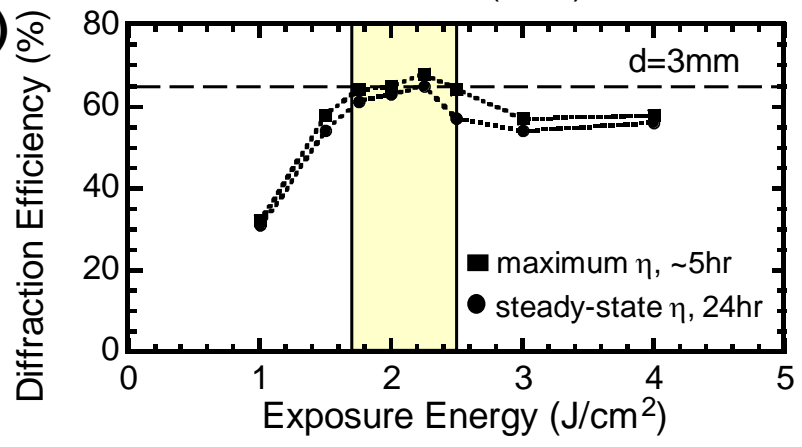

Figure 4 


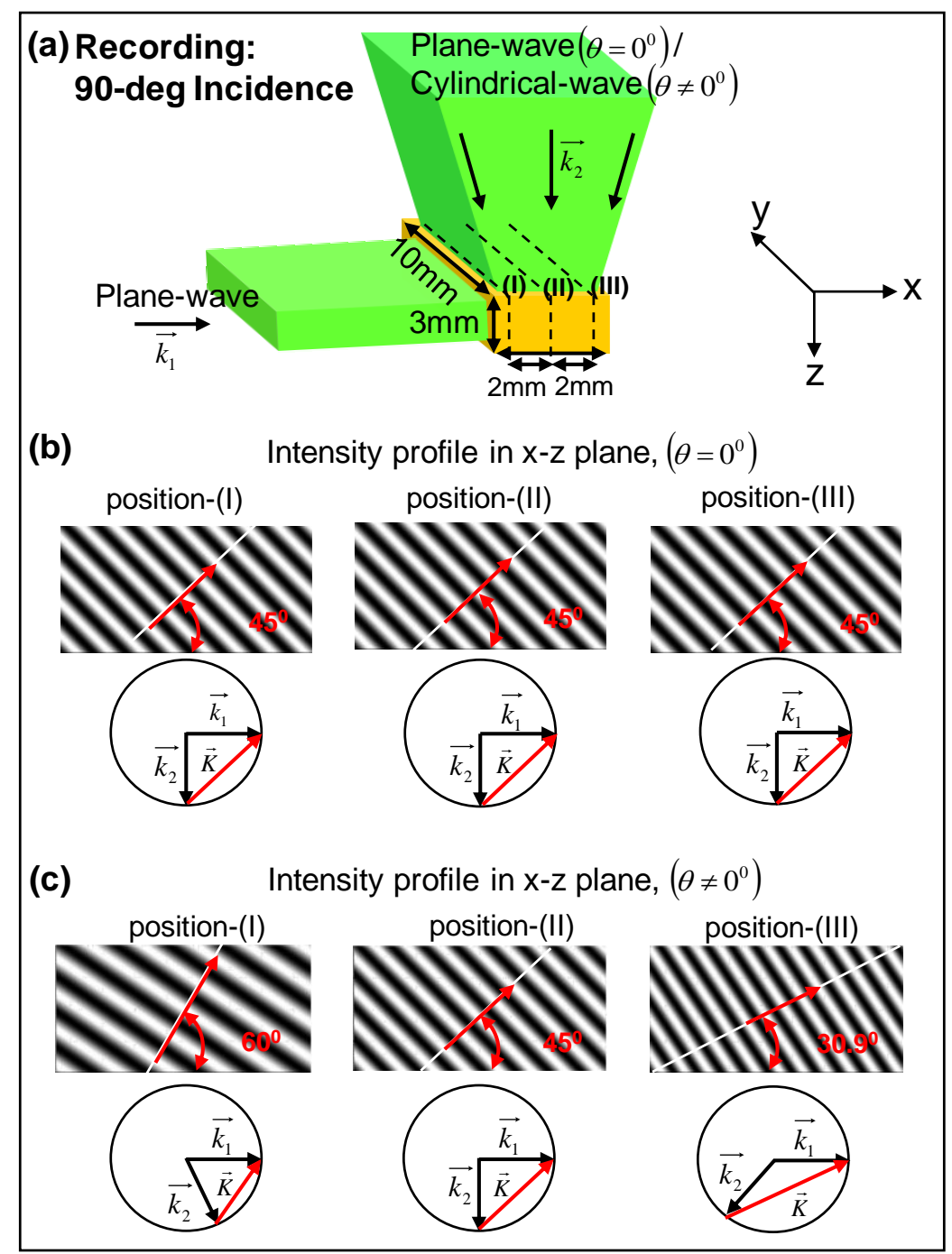

Figure 5 


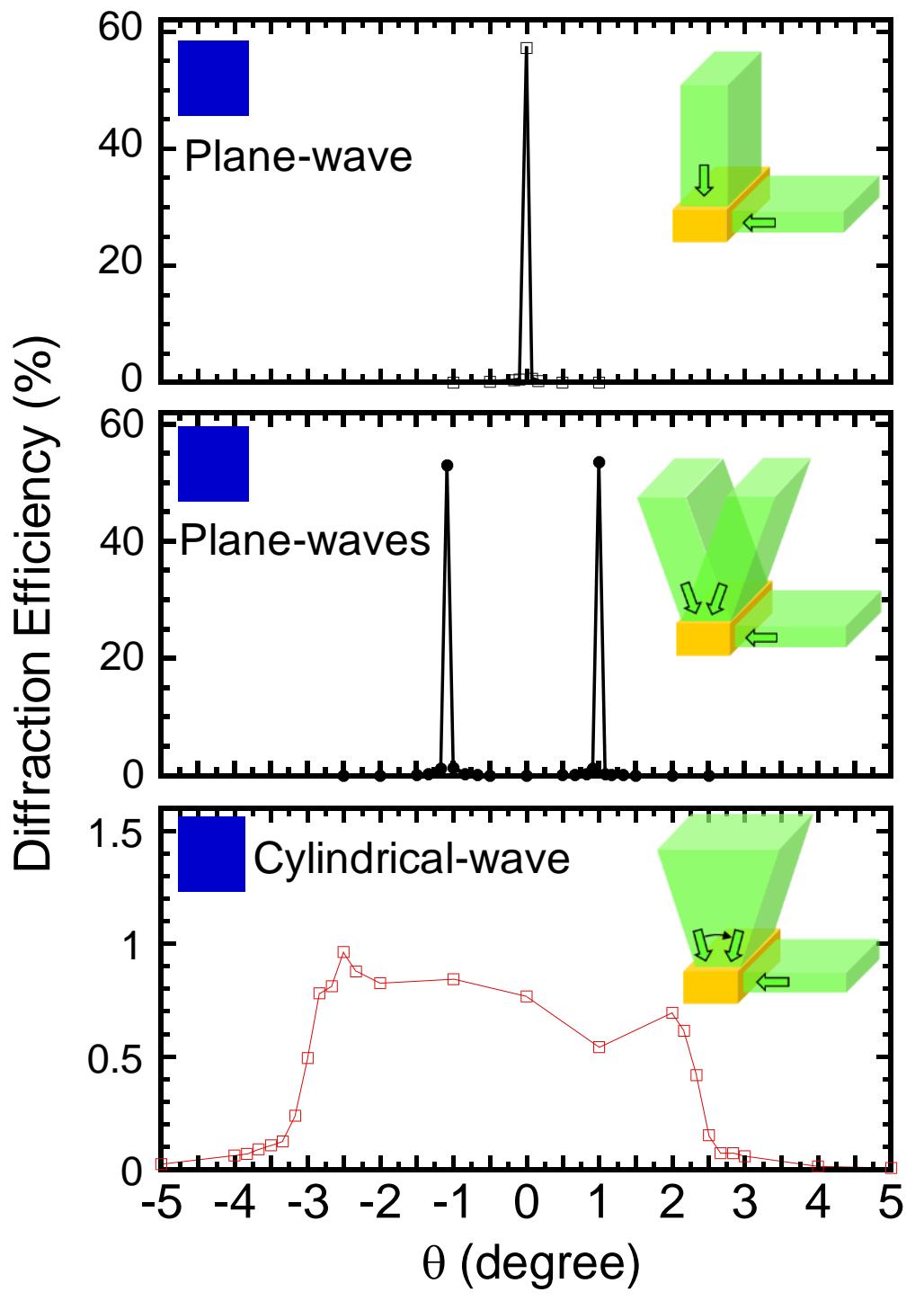

Figure 6 